\title{
High-pressure phase transformation in scandium
}

\author{
Y. K. Vohra, ${ }^{*}$ W. Grosshans, and W. B. Holzapfel \\ Universität - Gesamthochschule - Paderborn, Experimentalphysik, 4790 Paderborn, Federal Republic of Germany
}

(Received 6 November 1981; revised manuscript received 9 December 1981)

\begin{abstract}
The $3 d$ transition metal scandium $(\mathrm{Sc})$ has been studied under pressures up to $35 \mathrm{GPa}$ by energy-dispersive $\mathrm{x}$-ray diffraction. A structural phase transition was observed around $20 \mathrm{GPa}$ from hcp to $\beta$-Np structure (tetragonal, four atoms per unit cell). This high-pressure structure of $\mathrm{Sc}$ is different from any known structure of trivalent rare-earth metals and points out the limitations of canonical band-theory calculations which qualitatively accounted so far for the crystal-structure trends in the $d$-band metals, including lanthanides.
\end{abstract}

The group-IIIB transition metals scandium (Sc) and yttrium (Y) are usually grouped together with lanthanide metals to form the rare-earth series. The dependence of crystal structure of the regular trivalent lanthanide metals on atomic number, alloying, and pressure is closely interrelated. These metals show the rare-earth crystal-structure sequence $\mathrm{hcp} \rightarrow \mathrm{Sm}$-type $\rightarrow$ dhcp $\rightarrow$ fcc with decreasing atomic number and increasing pressure. ${ }^{1-3}$ The metal $Y$ is most similar to heavier lanthanides, ${ }^{2}$ alloyed with light lanthanides it exhibits the rare-earth crystalstructure sequence. In a recent experiment, it was observed that the rare-earth crystal-structure sequence appears also in $\mathrm{Y}$ with increasing pressures. ${ }^{4}$ This result shows that one does not need $f$ electrons to go through the rare-earth crystal-structure sequence but the bonding contribution of the $d$ electrons ( $U_{d}^{\text {bond }}$ ) is most dominant. In fact, canonical band-theory calculations by Duthie and Pettifor ${ }^{5}$ reproduced the stability of the various rare-earth crystal structures by a variation of the $d$-band occupancy from $N_{d}<1.7$ for hcp, to $1.7<N_{d}<2.3$ for Sm type, $2.3<N_{d}<2.6$ for dhcp, and to $N_{d}>2.6$ for fcc. Similarly, certain critical values of the ratio between the Wigner-Seitz radius and the ionic radius were earlier considered ${ }^{2,3}$ to characterize the stability ranges of the various rare-earth crystal structures, showing a close relation between $d$-band occupancy and this radius ratio. From this point of view, the present study was intended to look for the same structural sequence in Sc under pressure, since this radius ratio of 2.0 for $\mathrm{Sc}$ is close to the value of 1.91 for $\mathrm{Y}$ and to the range of values from 1.80 to 1.93 for heavy lanthanides. Also, in view of canonical band-theory calculations and considering an increase in number of $d$ electrons under pressure, $\mathrm{Sc}$ is similar to $\mathrm{Y}$ and heavy lanthanides.

The metal Sc like Y crystallizes in hcp structure (Sc I) at ambient conditions and goes to bcc structure ${ }^{6}$ at high temperatures (Sc II). Earlier high-pressure resistivity measurements ${ }^{7,8}$ at room temperature show resistance anamolies around $17 \mathrm{GPa}$ and led to the assumption of a structural phase transition at this pressure. Also, high-pressure low-temperature studies $^{7,8}$ show the onset of superconductivity in the same pressure region with superconducting transition temperature $\left(T_{c}\right)$ increasing with increasing pressure. The shock-wave studies ${ }^{9}$ on Sc and $\mathrm{Y}$ also provide indirect evidence of a low-pressure (10-20 GPa) phase transition. In the present investigations, Sc was studied by energy-dispersive $x$-ray diffraction (EDXD) using a diamond anvil cell with ruby manometer ${ }^{10}$ with the calibration constant $d \lambda / d P=3.65 \AA / \mathrm{GPa}$. Figure 1 shows the diffraction pattern of the hcp phase at $6.5 \mathrm{GPa}$. Around $20 \mathrm{GPa}$, a crystallographic phase transition was noticed with new features in the diffraction pattern. This transition was only complet-

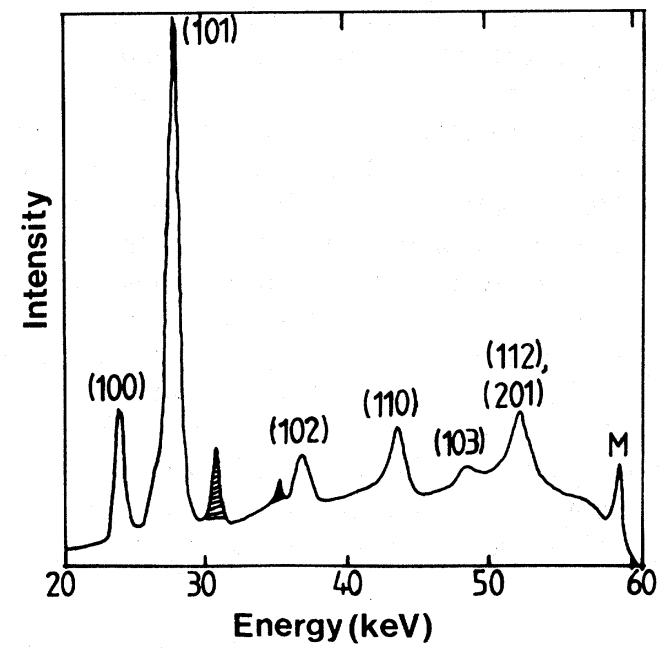

FIG. 1. EDXD pattern of Sc I (hcp) at $6.5 \mathrm{GPa}$ with increasing pressure. The shaded lines are the extraneous Baflourescence lines and $M$ is the marker for energy calibration. (002) peak is not observed because of basal plane texture. 


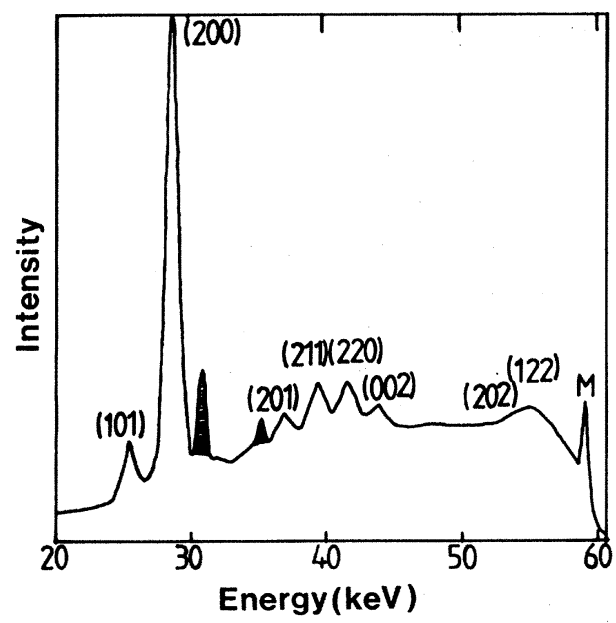

FIG. 2. EDXD pattern of Sc III at $33.5 \mathrm{GPa}$. The diffraction pattern was indexed to a tetragonal lattice with $c / a=0.673$.

ed at around $26 \mathrm{GPa}$. The diffraction pattern of this new phase ${ }^{11}$ (Sc III) at $33.5 \mathrm{GPa}$ is shown in Fig. 2. Among the cubic, tetragonal, and hexagonal crystal systems, only a tetragonal lattice $[a=473(2) \mathrm{pm}$, $c=318(1) \mathrm{pm}]$ with axial ratio of $c / a=0.673(5)$ could be fitted to this diffraction pattern. The average deviation of the observed diffraction-line positions from the calculated one was about $0.5 \%$. Reasonable atomic volumes and a volume decrease of $\sim 1 \%$ at $22.5 \mathrm{GPa}$ are obtained for 4 atoms per unit cell. The observed $c / a$ ratio and intensity ratios are similar to the high-temperature phase of neptunium ( $\beta$-Np, space group $\left.D_{4 h}^{7} P 4 / n m m\right)$. On unloading, this Sc III phase is retained down to around 18 GPa and back transformation to hcp phase was completed at about $14 \mathrm{GPa}$. The back-transformed hcp phase at $9.8 \mathrm{GPa}$ is shown in Fig. 3. The original basal plane texture of the hcp phase is destroyed after cycling through structural phase transitions. This is clearly seen by the appearance of the (002) reflection in Fig. 3 in comparison to Fig. 1 as well as by the increase in intensity of $(h k l)$ reflections with large $l$ values. In comparison with the earlier resistivity study on Sc under pressure, ${ }^{8}$ a later onset of the transition and a larger hysteresis are observed, probably because of smaller nonhydrostatic stress and other

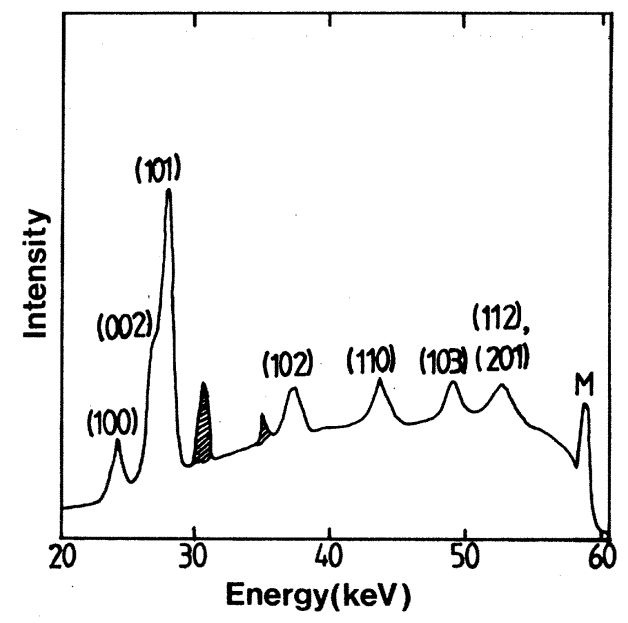

FIG. 3. EDXD pattern of back-transformed Sc I (hcp) at $9.8 \mathrm{GPa}$ taken on decreasing pressure. Note that the original texture in the hcp phase is destroyed as a result of cycling through the phase transformation.

kinetics factors.

The present results of the high-pressure study on $\mathrm{Sc}$ demonstrate that $\mathrm{Sc}$ does not go through the rareearth crystal-structure sequence that has been observed for $\mathrm{Y}$ and the other trivalent lanthanides. So, the rare-earth crystal-structure sequence observed in $5 d$-metals ${ }^{1,12} \mathrm{La}-\mathrm{Lu}$ and the $4 d$ metal $^{4} \mathrm{Y}$ is not observed in the $3 d$ metal Sc. This fact indicates that a simple canonical band-theory calculation ${ }^{5}$ which neglects $s-d$ hybridization and compares only the canonical $d$-band energies is not enough to explain this crystal-structure sequence. One has to include the effects of crystal potentials, atomic volumes and bands hybridization explicitly and not merely through the change in $d$-band occupancy. Therefore, accurate calculation of the total energy for different crystal structures going beyond atomic sphere approximation $^{13}$ may be required to explain the high-pressure behavior of Sc.

\section{ACKNOWLEDGMENTS}

One of us (Y. K. V.) was supported by the Alexander von Humboldt Foundation.
"Permanent address: Neutron Physics Division, Bhabha Atomic Research Centre, Trombay, Bombay - 400085, India.

${ }^{1}$ A. Jayaraman and R. C. Sherwood, Phys. Rev. 134, A691 (1964).
2B. Johansson and A. Rosengren, Phys. Rev. B 11, 2836 (1975).

${ }^{3 B}$. Johansson, in Rare Earths and Actinides - 1977, edited by W. D. Corner and B. K. Tanner, Inst. Phys. Conf. Ser. No. 37 (IOP, London, 1978), Chap. 3, p. 39. 
${ }^{4}$ Y. K. Vohra, H. Olijnyk, W. Grosshans, and W. B. Holzapfel, Phys. Rev. Lett. 47, 1065 (1981).

5J. C. Duthie and D. G. Pettifor, Phys. Rev. Lett. $\underline{38}, 564$ (1977).

${ }^{6}$ B. J. Beaudry and K. A. Gschneidner, Jr., in Handbook on the Physics and Chemistry of Rare Earths, edited by K. A. Gschneidner and L. Eyring (North-Holland, Amsterdam, 1978), Vol. I, p. 173.

${ }^{7} \mathrm{C}$. Probst and J. Wittig, in Handbook on the Physics and Chemistry of Rare Earths, edited by K. A. Gschneidner and L. Eyring, (North-Holland, Amsterdam, 1978), Vol. I, p. 749.

8J. Wittig, C. Probst, F. A. Schmidt, and K. A. Gschneidner,
Jr., Phys. Rev. Lett. 42, 469 (1979).

${ }^{9}$ W. J. Carter, J. N. Fritz, S. P. Marsh, and R. G. McQueen, J. Phys. Chem. Solids 36, 741 (1975).

${ }^{10} \mathrm{~W}$. B. Holzapfel and W. May, in High Pressure Research in Geophysics, edited by S. Akimoto and M. H. Manghnani (Centre of Academic Publications, Japan, 1981).

${ }^{11} \mathrm{~A}$ different numbering to identify phases with respect to Ref. 8 is used to include the high-temperature bcc phase as Sc II.

${ }^{12}$ L. Liu, J. Phys. Chem. Solids $\underline{36}, 31$ (1975).

${ }^{13} \mathrm{~A}$. R. Mackintosh and O. K. Andersen, in Electrons at the Fermi Surface, edited by M. Springford (Cambridge University Press, Cambridge, England, 1980), Chap. 5. 